Mater. Res. Soc. Symp. Proc. Vol. 1426 @ 2012 Materials Research Society

DOI: 10.1557/opl.2012.1094

\title{
Low Temperature Plasma Synthesis of Nanocrystals and their Application to the Growth of Crystalline Silicon and Germanium Thin Films
}

\author{
P. Roca i Cabarrocas ${ }^{1}$, K.H. Kim ${ }^{1,2}$, R. Cariou ${ }^{1}$, M. Labrune ${ }^{1,2}$, E.V. Johnson ${ }^{1}$, M. Moreno ${ }^{1}$, A. \\ Torres Rios ${ }^{1}$, S. Abolmasov ${ }^{1}$, and S. Kasouit ${ }^{1,2}$ \\ ${ }^{1}$ LPICM, CNRS, Ecole Polytechnique, 91128 Palaiseau, France \\ ${ }^{2}$ Total S.A., Gas \& Power - R\&D Division, Courbevoie, France
}

\begin{abstract}
We summarize our research studies on the synthesis of silicon and germanium nanocrystals and their application to the growth of a variety of thin films, spanning the range from fully disordered amorphous up to fully ordered crystalline. All these films are deposited in a standard radio-frequency glow discharge system at low temperature $\left(\sim 200^{\circ} \mathrm{C}\right)$. We show how the plasma synthesis of silicon nanocrystals, initially a side effect of powder formation, has become over the years an exciting field of research which has opened the way to new opportunities in the field of materials deposition and their application to optoelectronic devices. Our results suggest that epitaxy requires the melting/amorphization of the nanocrystals upon impact on the substrate, the subsequent epitaxial growth being favored on (100) c-Si substrates. As a consequence, the control of the impact energy is a critical aspect of the growth which will require new strategies such as the use of tailored voltage waveforms.
\end{abstract}

\section{INTRODUCTION}

Plasma Enhanced Chemical Vapor Deposition (PECVD) based on parallel plate capacitively coupled RF glow discharge systems has become the mainstream technology for large area deposition of hydrogenated amorphous $(\mathrm{a}-\mathrm{Si}: \mathrm{H})$ and microcrystalline silicon $(\mu \mathrm{c}-\mathrm{Si}: \mathrm{H})$ thin films. It has allowed the development of flat panel displays and tandem a-Si:H/ $\mu \mathrm{cSi}: \mathrm{H}$ solar cells [1]. The standard model to describe the process leading to low defect density a-Si:H and $\mu \mathrm{c}-$ $\mathrm{Si}: \mathrm{H}$ films is based on the surface diffusion of silicon radicals, in particular $\mathrm{SiH}_{3}[2,3]$. However, when trying to increase the deposition rate by tuning process parameters such as the RF power, the total pressure, the dilution of silane, the excitation frequency,...the common issue is the formation of powders $[4,5]$. This has been an active subject of research, mostly aiming at avoiding powder and cluster formation [6]. Yet, numerous groups have reported on the formation of silicon nanocrystals in silane discharges $[7,8,9]$. Moreover, we have discovered that silicon nanocrystals produced in $\mathrm{SiH}_{4}-\mathrm{H}_{2}$ gas mixtures can be incorporated in the growing film, leading to a nanostructured material that we have called hydrogenated polymorphous silicon (pm-Si:H) [10]. Interestingly, pm-Si:H films display improved transport properties and stability with respect to a-Si:H $[11,12]$ which makes them attractive for stable solar cells and thin film transistors [13,14]. This approach has been further extended to $\mathrm{SiF}_{4}-\mathrm{H}_{2}-\mathrm{Ar}$ gas mixtures [15] leading to the deposition of microcrystalline silicon films, as well as to $\mathrm{GeH}_{4}-\mathrm{H}_{2}-\mathrm{Ar}$ gas mixtures [16] for germanium nanocrystals synthesis.

In this paper we review some results concerning the plasma synthesis of nanocrystals, their importance for the deposition of silicon thin films and the related plasma/surface interactions, that have to be controlled in order to achieve materials with optimized optoelectronic properties. In particular we highlight the process conditions that, depending on the 
nature of the substrate, can lead to the growth of either polymorphous silicon on glass substrates or epitaxial films on (100) c-Si substrates. Moreover, the flexibility provided by the PECVD process allows us to produce $\mathrm{Si} / \mathrm{Ge}$ quantum wells. In our opinion, with further development PECVD reactors will become a universal toolbox for the deposition of a wide range of thin film materials.

\section{EXPERIMENTS}

Silicon and germanium thin films were deposited using standard RF glow discharge in a multiplasma-monochamber deposition system [17]. This type of parallel plate capacitively coupled reactor has been developed to allow uniform deposition of silicon thin films over areas larger than $5 \mathrm{~m}^{2}$ in GEN 10 production tools. As indicated above, the standard approach to explain a-Si:H and $\mu \mathrm{c}-\mathrm{Si}: \mathrm{H}$ deposition is to consider the contribution of $\mathrm{SiH}_{\mathrm{x}}$ radicals (in particular $\mathrm{SiH}_{3}$ ) and atomic hydrogen. However, as shown in Figure 1, there are many more species than radicals which can interact with the substrate and contribute to thin film deposition. For example, the bombardment of the surface by positive ions has been largely explored and recognized as a source of defect creation in a-Si:H thin films [18] and of amorphization in the case of $\mu \mathrm{c}-\mathrm{Si}: \mathrm{H}$ deposition [19].

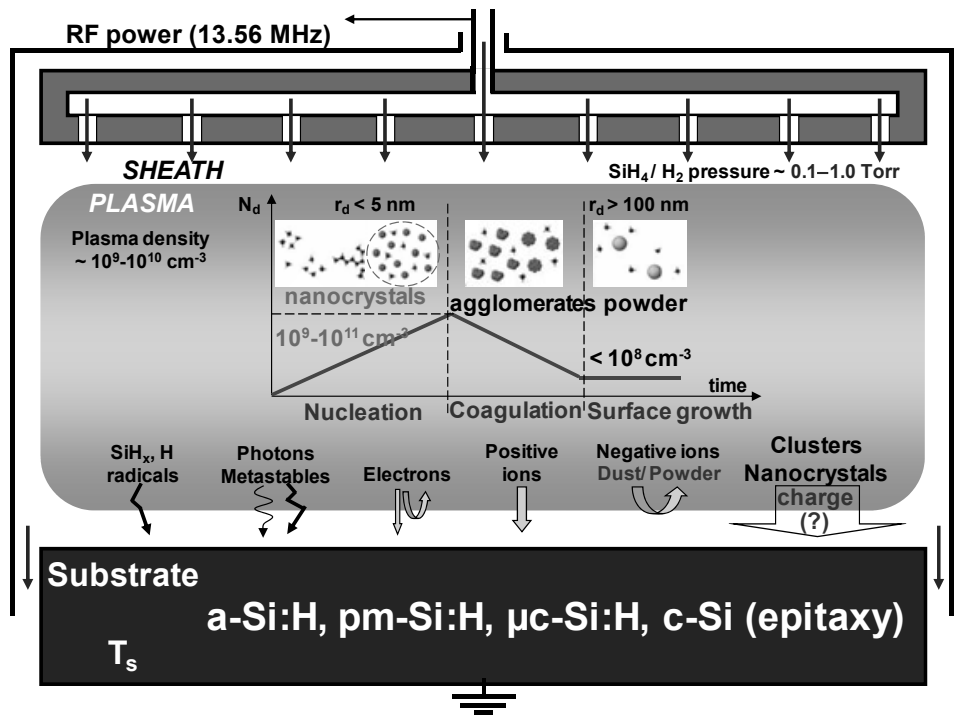

Figure 1. Schematic diagram of a parallel plate capacitively coupled RF glow discharge reactor. The plasma processes are schematically depicted in the bulk of the plasma, while the species contributing to deposition are shown at the plasma sheath. Depending on the type of precursor a wide range of thin films can be obtained, from standard a-Si:H to ordered epitaxial films. 
Although at low silane dissociation rates silicon radicals and ions can be considered to be the main film precursors, increasing the deposition rate will lead to secondary reactions and the formation of clusters, nanocrystals and eventually powders $[5,20]$. The genesis of powder formation is schematically described in the inset of figure 1. It develops through three phases: i) nucleation and accumulation, ii) coagulation or agglomeration and iii) surface growth $[4,21]$. It is interesting to note that the incubation and nucleation phase proceeds until a critical density of nanoparticles (of radius smaller than $\sim 5 \mathrm{~nm}$ ) is reached $\left(\sim 10^{10}-10^{11} \mathrm{~cm}^{-3}\right)$ and that this density exceeds by one or two orders of magnitude the electron density. This implies that a large fraction of nanoparticles must be neutral or positively charged and therefore they can contribute to deposition. Once the critical density is reached the agglomeration phase will start, leading to an increase in the particles size and a dramatic decrease in their density $\left(<10^{8} \mathrm{~cm}^{-3}\right)$. Such transition, known as the $\alpha-\gamma^{\prime}$ transition [22], manifests itself by strong changes in the plasma properties (electron density, temperature,...) due to the fact that large particles/powders become negatively charged and therefore trapped within the plasma bulk by the sheath potential. Our main focus is on the process conditions leading the formation of silicon clusters and nanocrystals, while avoiding the coagulation phase. This can be achieved by the use of modulated discharges [23] or by adjusting the process conditions in such a way that the loss rate of nanoparticles to the surface is higher than their production rate. In other words, one can adjust the process conditions in order to keep the plasma in the nucleation regime at steady-state.

\section{DISCUSSION}

While the general consensus on the deposition of amorphous and microcrystalline silicon thin films relies on $\mathrm{SiH}_{3}$ radicals being the most important species for obtaining device grade material, we have been focusing over the past fifteen years on promoting gas phase reactions that result in the formation of nanocrystals. The question must be asked, why would one want to deliberately use more complex process conditions? Besides the increase in deposition rate, we demonstrate in the following section the main benefits of using silicon nanocrystals, with a particular emphasis on the obtaining of epitaxial growth of silicon and germanium films at low substrate temperature. Then we will move to the critical issue of plasma/surface interactions with a particular emphasis on the effects of ion bombardment energy and how to control it. We will conclude with the perspectives opened by this new approach to thin film deposition.

\section{Nanocrystals as ideal building blocks for thin film deposition}

Plasma synthesis of clusters and nanocrystals in low pressure PECVD reactors has been largely studied over the past ten years. Indeed, it has been shown that such nanocrystals can be produced in a controlled manner is various types of reactors $[7,8,9]$ and that they can be produced with quite a narrow size dispersion. Moreover, it has been shown that their radial growth rate can be extremely high (up to $75 \mathrm{~nm} / \mathrm{s}$ ) [23] when compared to typical thin film deposition rates $(0.1-1 \mathrm{~nm} / \mathrm{s})$. Quantum size effects associated with the nanocrystals have motivated their application as light sources in electroluminescent devices by inserting them in organic or inorganic matrix materials [24,25]. Our group has pursued the route of using the plasma synthesized nanocrystals as building blocks for thin film deposition. In this way we have demonstrated that we can increase both the deposition rate and the efficiency of PIN solar cells and modules [26] in which the intrinsic layer is made of pm-Si:H. 
This approach has been extended to the growth of hydrogenated microcrystalline silicon $(\mu \mathrm{c}-\mathrm{Si}: \mathrm{H})$ films. Indeed, $\mu \mathrm{c}-\mathrm{Si}: \mathrm{H}$ is a complex material whose growth process involves surface and subsurface reactions of silicon radicals and atomic hydrogen. Such process develops through four phases (incubation, nucleation, growth and steady-state) which limit the deposition rate of the material [27]. However, as silicon nanocrystals can be produced at high rate in the plasma, why not to use them to promote $\mu \mathrm{c}-\mathrm{Si}: \mathrm{H}$ growth? In this way the challenging (when taking place on the substrate) incubation and nucleation phases can be short-circuited and $\mu \mathrm{c}-\mathrm{Si}: \mathrm{H}$ growth can start from the beginning of deposition. Using this approach we have been able to fabricate bottom gate thin film transistors without any amorphous incubation layer [28].

While pm-Si:H and $\mu \mathrm{c}-\mathrm{Si}: \mathrm{H}$ films are interesting alternatives/complements to a-Si:H, it would be interesting to grow crystalline silicon films using the same equipment. In fact, many groups have reported on epitaxial growth of silicon films on (100) c-Si substrates. It is well known in the heterojunction solar cells community that an epitaxial or mixed phase material (a$\mathrm{Si}: \mathrm{H}$ and c-Si) cannot provide the required surface passivation [29]. However, it has been shown recently that a thin epitaxial layer in between a c-Si wafer and an a-Si:H layer produces enhanced field effect passivation and shows promising results [30]. Also, this epitaxy phenomenon can be desired to develop the so called wafer equivalent approach. High quality silicon epitaxy can be grown by $\mathrm{HWCVD}$ around $700^{\circ} \mathrm{C}$ with $\mathrm{SiH}_{3}$ and $\mathrm{Si}_{2} \mathrm{H}_{2}$ being the main radicals involved [31]. But when trying to promote epitaxial growth at low temperature, the hydrogen rich environment of the PECVD or HWCVD process leads to epitaxy break-down which prevents the growth of thick layers [32]. Nevertheless, we have demonstrated that the use of process conditions leading to pm-Si:H growth on glass results in epitaxial growth on (100) c-Si substrates [33]. This is illustrated in Figure 2 where we present the deposition rate and composition of films deposited on (100) c-Si substrates in a RF glow discharge reactor [17]. The substrate temperature was kept at $175{ }^{\circ} \mathrm{C}$, the hydrogen flow rate at $500 \mathrm{sccm}$, the total pressure at 2.9 mbarr and the RF power density at $68 \mathrm{~mW} / \mathrm{cm}^{2}$. The properties of the films were deduced from the modeling of the spectroscopic ellipsometry measurements (UVISEL from HORIBA Jobin-Yvon).

As shown in figure 2, the deposition rate increases linearly with the silane flow rate. However the properties of the films are strongly dependent on the nature of the substrate. As indicated on the bottom of the figure, the structure of the films deposited on glass and (111) c-Si substrates progressively changes from $\mu \mathrm{c}-\mathrm{Si}: \mathrm{H}$ at low silane flow rates to $\mathrm{pm}-\mathrm{Si}: \mathrm{H}$ at intermediate values of the silane flow rate, corresponding to plasma conditions where silicon nanocrystals are formed in the plasma. Finally, at high silane flow rates we speculate that the agglomeration of nanocrystals leading to powder formation acts as a sink for the nanocrystals which cannot contribute anymore to deposition. Under powder conditions only long-lived radicals (low sticking coefficient) such as $\mathrm{SiH}_{3}$ can contribute to deposition and the film grows as a-Si:H [20]. It is interesting to note that for films co-deposited on glass, c-Si (100) and c-Si (111) we obtain the same deposition rate.

Looking now at the composition of the films deposited on c-Si (100), figure 2 shows that if silane flow rate is increased the fraction of mono c-Si increases while that of large grain polySi material decreases, in other words, the film becomes monocrystalline as viewed by spectroscopic ellipsometry. However at $50 \mathrm{sccm}$ the film turns to be heterogeneous consisting of $\mathrm{c}-\mathrm{Si}$ and amorphous phases. On the other hand, for silane flow rates lower than $30 \mathrm{sccm}$ the films are $\mu \mathrm{c}-\mathrm{Si}: \mathrm{H}$, with an important fraction of large grains. Interestingly enough, for a silane flow rate in the range of $35-45 \mathrm{sccm}$ the films are monocrystalline silicon, thus suggesting an epitaxial growth. 


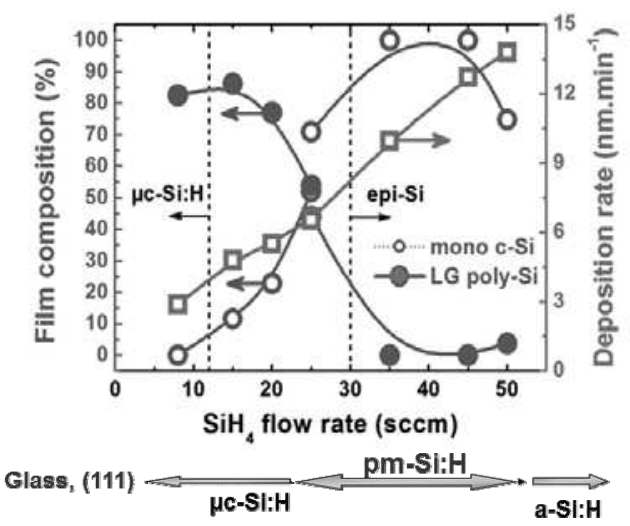

Figure 2. Deposition rate and composition of the films deposited on (100) c-Si substrates as functions of the silane flow rate. The bottom part of the figure shows the effect of the substrate on the structure of the films for the same process conditions. Lines are provided as a guide to the eye.

Epitaxial growth is supported by high resolution transmission electron microscopy measurements performed in a JEOL $2200 \mathrm{FS}$ microscope. Figure 3 gives an example of a $\mathrm{Ge} / \mathrm{Si}$ multilayer structure grown on a (100) GaAs wafer [34]. The figure shows the excellent quality of the interface between the GaAs substrate and the first $20 \mathrm{~nm}$ thick Ge layer, followed by the first Si epitaxial layer.

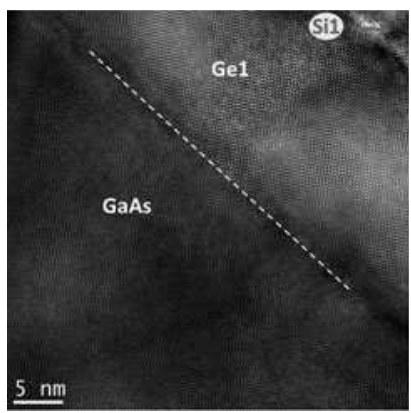

Figure 3. High resolution TEM image of the first Ge and Si layers from a multilayer stack deposited on a (100) GaAs substrate at $175^{\circ} \mathrm{C}[34]$. 
These results demonstrate that epitaxial growth is possible by PECVD at low temperatures. Moreover, by making heterojunction solar cells using $3 \mu \mathrm{m}$ thick epitaxial silicon layers as the absorber, we have demonstrated that the electronic quality of the films is suitable for solar cell applications [35]. However these results do not tell us how epitaxial growth takes place in such PECVD environment, nor why epitaxy only takes place in a narrow range of process conditions.

\section{Plasma/surface interactions}

As discussed above the substrate plays a crucial role in the structure of the films. This is a quite well known effect, in particular for the case of $\mu \mathrm{c}-\mathrm{Si}: \mathrm{H}$ films [27]. However, the most striking result is the observation of epitaxial growth on a (100) c-Si substrate at such low temperature in a hydrogen rich environment. In order to understand this result, one must look at plasma/surface interactions under complex process conditions, such as those depicted in figure 1 . Our working hypothesis to explain epitaxial growth is based on the presence of silicon nanocrystals in the plasma and their contribution to deposition. So the question is: how do the nanocrystals interact with the substrate?

To answer this question we need to know what is the electric charge on the nanocrystals in question. In the case of neutral particles, it is obvious that they will diffuse to the substrate through the sheath, as for $\mathrm{SiH}_{\mathrm{x}}$ radicals. On the contrary, if nanocrystals are negatively charged, they would be confined in the plasma by the plasma sheath potential (see figure 1) and would not be able to contribute to deposition. On the other hand, if they were positively charged, then they would be accelerated towards the substrate with an energy equal or smaller than the plasma potential. Under our process conditions ( $\sim 2 \mathrm{mbarr})$ the sheath is collisional and therefore positively charged particles will lose a part of their energy. We have previously demonstrated that nanocrystals contribute to deposition and that a non negligible fraction must be positively charged [36]. For this demonstration we adopted a triode configuration where the substrate, cooled by liquid nitrogen, was placed behind a mesh in order to be able to bias the substrate without affecting the plasma. In this way, one can increase or decrease the impact energy of the positively charged species by applying a negative or a positive bias to the substrate respectively. The use of a liquid nitrogen-cooled substrate aims at freezing the chemical reactions which could lead to $\mu \mathrm{c}-\mathrm{Si}: \mathrm{H}$ growth via surface and subsurface reactions [27]. This ensures that the crystalline fraction, deduced from Raman spectroscopy measurements, is due to the nanocrystals produced in the plasma which reach the substrate when neutral (by diffusion) or positively charged (being accelerated by the plasma potential). A series of films was deposited under the same conditions while only varying the bias applied to the substrate. Table I shows the crystalline fraction of the films as a function of the bias.

Table I. Effect of the substrate bias on the crystalline fraction of silicon thin films deposited in a triode system with the substrate cooled by liquid nitrogen.

\begin{tabular}{|l|l|l|l|l|l|l|l|}
\hline Substrate Bias (Volts) & -50 & -25 & 0 & 25 & 50 & 75 & 100 \\
\hline Cryst. Fraction (\%) & 0 & 0 & 26 & 100 & 50 & 35 & 25 \\
\hline
\end{tabular}

The results in Table I clearly demonstrate the importance of the impact energy of the nanocrystals on the structure of the films. Indeed, if the impact energy is too high (this is the case 
of applying a bias voltage of -50 and $-25 \mathrm{~V}$ ), then the nanocrystals amorphize upon impact. This has been studied by modeling and for example it has been suggested that nanocrystals with impact energies higher than $0.5 \mathrm{eV} /$ atom can amorphize [37].

Table I also shows that there is a saturation of the crystalline fraction for values of the bias in the range of 75 to $100 \mathrm{~V}$. This indicates that the nanocrystals contributing to deposition at such high values of the bias voltage are neutral, the positively charged ones being repelled from the substrate by the bias. On the other hand at $25 \mathrm{~V}$ we obtain a crystalline fraction of $100 \%$, which suggests that more than $75 \%$ of the nanocrystals contributing to growth should be positively charged. This estimation is consistent with independent measurement of the contribution of positively charged particles to the growth [38].

These results highlight the importance of controlling the impact energy of the nanocrystals, along with the ions. While this can be achieved in various ways (such as the use of triode systems as described above, moving to VHF plasmas or microwave plasmas with lower plasma potential, or higher process pressures), the recently developed Tailored Voltage Waveform (TVWF) approach is an elegant way of fine tuning the plasma potential without changing the reactor design. Using non-sinusoidal RF waveforms (in fact composed of multiple harmonics, as shown in Figure 4, for a base frequency of $15 \mathrm{MHz}$ ), allows one to trade off the sheath voltage drop at the substrate with that at the RF-electrode [39]. By doing so, one can decouple the ion-bombardment energy at the substrate from the injected RF power. Using this technique for $\mu \mathrm{c}-\mathrm{Si}: \mathrm{H}$ deposition, one can control the crystalline fraction in the material as measured by spectroscopic ellipsometry and Raman spectroscopy (as shown in Fig. 4b) simply by inverting the shape of the waveform. Recently, it has been demonstrated that material deposited using the "Peaks" wave function gives good device quality when used as the absorbing layer in a PIN solar cell [40]. Most interestingly, this technique can be applied to existing PECVD reactors with no modifications to the vacuum chamber itself.
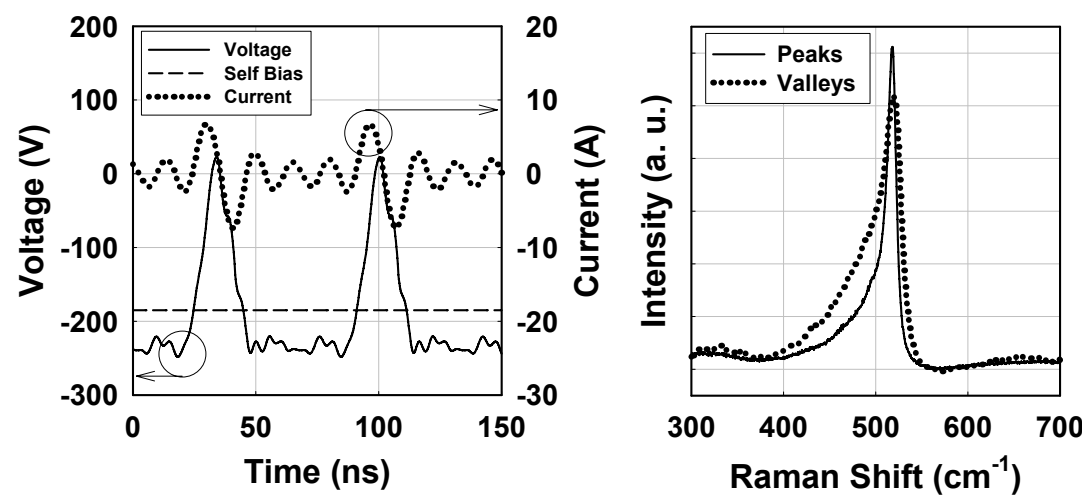

Figure 4. Peaks tailored voltage waveforms measured at the RF feedthrough during a PECVD process. The "Peaks" waveform reduces the ion bombardment energy at the substrate, whereas the "Valleys" waveform enhances it. This effect can be seen on the Raman spectra (right) where the peaks waveform results in a sharper TO crystalline peak, with lower amorphous fraction, characterized by the shoulder at $\sim 480 \mathrm{~cm}^{-1}[39]$. 


\section{Epitaxial growth. Do we need nanocrystals?}

So far, we have seen that the ion bombardment energy is a crucial parameter controlling the properties of silicon thin films, in particular their crystallinity. In the deposition of pm-Si:H and $\mu \mathrm{c}-\mathrm{Si}: \mathrm{H}$ films, excessive impact energy will lead to the amorphization of the material. Molecular dynamics simulations have shown that at high impact energy $(>0.5 \mathrm{eV} /$ atom $)$, the clusters will break down upon impact on the surface [37]. This threshold situation may present itself in a typical PECVD system where for a positively charged nanocrystal of $2 \mathrm{~nm}$, consisting of $\sim 240$ atoms, à plasma potential of $100 \mathrm{~V}$ will provide and average energy of $0.4 \mathrm{eV} /$ atom for the positively charged nanocrystals. For a $1 \mathrm{~nm}$ nanocrystal ( $\sim 10$ atoms) the impact energy will be excessively high and the nanocrystal will have no chances of surviving the impact. In other words, only large nanocrystals can survive the impact and lead to pm-Si:H and $\mu \mathrm{c}-\mathrm{Si}: \mathrm{H}$ films [41]. However, the question is whether nanocrystals are required for epitaxial growth. Indeed, assuming that nanocrystals are the building blocks for epitaxial growth, then the crucial question is: how the nanocrystals can be accommodated on the (100) c-Si surface and produce a dense epitaxial film?

Looking back at the results of films co-deposited on glass, c-Si (111) and c-Si (100), we note that for given deposition conditions only the films on (100) are crystalline. As the film precursors are the same and independent of the substrate, the fact that we do not observe nano- or microcrystalline silicon deposition on glass or (111) c-Si substrates suggests that the silicon nanocrystals should be positively charged and are amorphized upon impact. Only the larger ones can survive and this leads to the small crystalline fraction $(<10 \%)$ detected in pm-Si:H films [39]. However, if the nanocrystals amorphize upon impact on glass and (111) c-Si substrates, this should also be the case on (100) c-Si. In other words the structure of the clusters (amorphous or crystalline) does not matter for the epitaxial growth, but only their impact energy. This is similar to epitaxial growth achieved by the ionized cluster-beam deposition technique [42], where ionized silicon clusters have been used to produce epitaxial films. Modeling of the process $[37,43]$ showed that this relies on the breakdown of the clusters upon impact. Of course, the detailed mechanism of the interaction between the charged nanoparticles and the substrate will require further studies, in particular to determine up to which point the clusters should break down or just amorphize to allow for a crystalline growth from the ordered crystalline substrate. The fact that the solid phase crystalline growth from amorphous silicon on c-Si (100) is about 25 times faster than on (111) in the temperature range of $450-575^{\circ} \mathrm{C}$ [44] could explain the observed lack of epitaxy on (111) substrates. As a conclusion epitaxial growth in a low temperature PECVD system can be explained by interaction of charged clusters (amorphous or crystalline) with a c-Si substrate and involves amorphization (partial melting) of the cluster upon impact. The temperature spike associated with the amorphization could provide the required energy for recrystallization of the cluster.

\section{PECVD as a versatile technique for thin film deposition: from amorphous to crystalline films}

The results presented above show that by tuning the PECVD process conditions from these of a radical-based deposition process towards a cluster- and nanocrystal-based one opens new possibilities for a wide range of materials, as summarized in figure 5. While a-Si:H remains the reference material for large area electronic applications, one would like to improve its 
electronic properties and stability. This is partly achieved with $\mu \mathrm{c}-\mathrm{Si}: \mathrm{H}$ thin films. In this search of materials spanning from disordered to ordered ones, the plasma synthesis of silicon and germanium nanocrystals (quantum dots) as well as the epitaxial growth of silicon and germanium multilayer structures (see Fig. 3) open new possibilities for extending the range of materials and devices (solar cells, thin film transistors, detectors, ...) that can be produced. A major result of our studies is that this wide range of materials can be achieved in the same PECVD reactor by simply changing the deposition paradigm from a radical assisted growth process towards a nanocrystal assisted one.

The full development of the opportunities summarized in figure 5 implies moving to more complex and challenging process conditions. The above results, while providing a proof of concept, call for further studies to better understand the various steps involved in thin film deposition. Concerning plasma processes, further work is required to control the size and density of clusters and nanocrystals. The main challenge is to find ways to increase the density of nanoparticles in the plasma while avoiding the agglomeration phase; i.e. to avoid them getting negatively charged. Furthermore, concerning the structure of the clusters, ab-initio molecular dynamic simulations suggest that hydrogen recombination on their surface is a key parameter for their crystallization [45]. However, as shown above, the critical issue for epitaxial growth is not the structure of the clusters but their impact energy. This encourages one to develop strategies to control the plasma potential independently of the energy coupled to the discharge, such as the use of TVWF technique.

\section{Materials Low temperature PECVD: Devices}

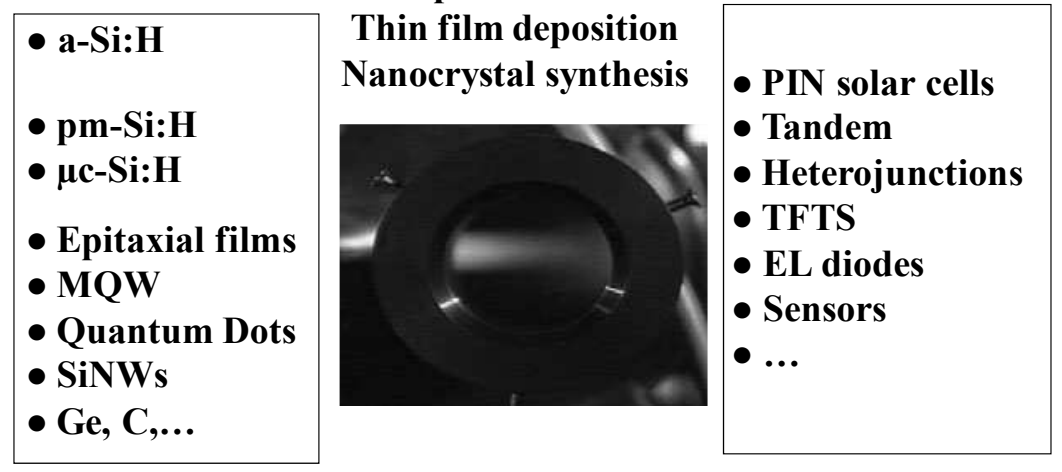

Figure 5. Plasma enhanced chemical vapor deposition as a versatile toolbox for the deposition of a wide range of materials and devices.

\section{CONCLUSIONS}

While the literature on plasma synthesis of silicon nanocrystals is quite rich, few groups have linked them to the deposition of thin films. Our pioneering work on hydrogenated polymorphous silicon has demonstrated the benefit of using plasma synthesized silicon nanocrystals as building blocks for materials with medium range order and better transport properties and stability compared to a-Si:H. This approach has been further extended to the case 
of epitaxial growth on (100) c-Si substrates. In this case it appears that the critical parameter is not the structure of the clusters but their impact energy which is the critical parameter as epitaxy proceeds from the recrystallization of amorphized clusters/nanocrystals. In conclusion, plasma enhanced chemical vapor deposition, a technique widely used for a-Si:H and $\mu \mathrm{c}-\mathrm{Si}: \mathrm{H}$ deposition in large area industrial reactors, appears as a promising tool for processing high quality materials and devices.

\section{ACKNOWLEDGMENTS}

This work has been partly supported by European Project Fast-Track. The research is currently being conducted in the framework of a joint research team between Total and CNRSLPICM. The authors thank G. Patriarche for TEM measurements.

\section{REFERENCES}

1. S. Wagner, Phys. Status Solidi A 207, 501 (2010).

2. A. Matsuda, K. Nomoto, Y. Takeuchi, A. Suzuki, A. Yuuki, and J. Perrin, Surf. Sci. 227, 50 (1990).

3. W. M. M. Kessels, A. H. M. Smets, D. C. Marra, E. S. Aydil, D. C. Schram, and M. C. M. van de Sanden, Thin Solid Films 383, 154 (2001).

4. Dusty Plasmas: Physics, Chemistry, and Technological Impacts in Plasma Processing. Wiley, New York (1999), A. Bouchole editor.

5. P. Roca i Cabarrocas, Y. Djeridane, Th. Nguyen Tran, E.V. Johnson, A. Abramov and Q.

Zhang. Plasma Phys. Control. Fusion 50, 124037 (2008).

6. Y. Watanabe, M. Shiratani, T. Fukuzawa, H. Kawasaki, Y. Ueda, S. Singh, and H. Ohkura. J. Vac. Sci. Technol. A 15, 995 (1996).

7. T. Ifuku, M. Otobe, A. Itoh, and S. Oda. Jpn. J. Appl. Phys. 36, 4031 (1997).

8. L. Mangolini, E. Thimsen, and U. Kortshagen. Nano Letters, 5, 655 (2005).

9. R. M. Sankaran, D. Holunga, R. C. Flagan, and K. P. Giapis. Nano Letters, 5, 537 (2005).

10. P. Roca i Cabarrocas, S. Hamma, S.N. Sharma, J. Costa, and E. Bertran. J. Non Cryst. Solids 227-230, 871 (1998).

11. C. Longeaud, J.P. Kleider, P. Roca i Cabarrocas, S. Hamma, R. Meaudre and M. Meaudre. J. Non Cryst. Solids 227-230, 96 (1998).

12. P. Roca i Cabarrocas, A. Fontcuberta i Morral and Y. Poissant. Thin Solid Films Vol. 403-404, 39 (2002).

13. Y. Poissant, P. Chatterjee, and P. Roca i Cabarrocas. J. Appl. Phys. 94, 7305 (2003).

14. M. Oudwan, O. Moustapha, A. Abramov, D. Daineka, Y. Bonnassieux, and P. Roca i

Cabarrocas. Phys. Stat. Solidi A 207, 1245 (2009).

15. Y. Djeridane, A. Abramov, and P. Roca i Cabarrocas. Thin Solid Films 515, 7451 (2007).

16. E.V. Johnson, G. Patriarche and P. Roca i Cabarrocas. Appl. Phys. Lett. 92, 103108 (2008).

17. P. Roca i Cabarrocas, J.B. Chévrier, J. Huc, A. Lloret, J.Y. Parey, and J.P.M. Schmitt. J. of Vac. Sci. and Technol. A9, 2331 (1991).

18. P. Roca i Cabarrocas, P. Morin, V. Chu, J. Conde, J.Z. Liu, H.R. Park, and S. Wagner. J. Appl. Phys. 69, 2942 (1991).

19. B. Kalache, A.I. Kosarev, R. Vanderhaghen, and P. Roca i Cabarrocas. J. Appl. Phys. 93, $1262(2003)$. 
20. P. Roca i Cabarrocas, Th Nguyen-Tran, Y. Djeridane, A. Abramov, E. Johnson and G. Patriarche. J. Phys. D: Appl. Phys. 40, 2258 (2007).

21. A. Fridman, L. Boufendi, T. Hbid, B. Potapkin, and A. Bouchoule, J. Appl. Phys. 79, 1303 (1995).

22. Ch. Böhm and J. Perrin. J. Phys. D: Appl. Phys. 24, 865 (1991).

23. Th. Nguyen-Tran, P. Roca i Cabarrocas and G. Patriarche. Appl. Phys. Lett. 91, 111501 (2007).

24. Kai-Yuan Cheng, R. Anthony, U. R. Kortshagen, R.J. Holmes. NanoLetters 10, 1154 (2010). 25. J. Wang, V. Suendo, A. Abramov, L. Yu and P. Roca i Cabarrocas. Appl. Phys. Lett. 97, $221113(2010)$.

26. Y. M. Soro, A. Abramov, M.E. Guenier-Farret, E.V. Johnson, C. Longeaud, P. Roca i Cabarrocas, and J.P. Kleider. J. Non Cryst. Solids 354, 2092 (2008).

27. P. Roca i Cabarrocas, chapter 5 in Physics and Technology of Amorphous-Crystalline

Heterostructure Silicon Solar Cells. Edited by W. G. J. H. M. van Sark, L. Korte and F. Roca. Springer (2011).

28. P. Roca i Cabarrocas, Y. Djeridane, V.D. Bui, Y. Bonnassieux and A. Abramov. Solid State Electronics 52, 422 (2008).

29. S. De Wolf and M. Kondo. Appl. Phys. Letters 90, 042111 (2007).

30. B. Hekmatshoar, D. Shahrjerdi, and D.K. Sadana, in Electron Devices Meeting (IEDM), 2011

IEEE International (2011), pp. 36.6.1 -36.6.4.

31. Ch. W. Teplin, K. Alberi, M. Shub, C. Beall, I. T. Martin, M. J. Romero, D. L. Young, R. C. Reedy, P. Stradins, and H. M. Branz. Appl. Phys. Lett. 96, 201901 (2010).

32. C.W. Teplin, D.H. Levi, E. Iwaniczko, K.M. Jones, J.D. Perkins, and H.M. Branz, J. Appl. Phys. 97, 103536 (2005).

33. P. Roca i Cabarrocas, R. Cariou and M. Labrune. J. Non Cryst. Solids (2012). In press doi:10.1016/j.jnoncrysol.2011.12.113.

34. M. Labrune, X. Bril, G. Patriarche, L. Largeau, O. Maugin and P. Roca i Cabarrocas. EPJ Photovoltaics (submitted).

35. R. Cariou, M. Labrune and P. Roca i Cabarrocas. Solar Energy Materials and Solar Cells. 95, 2260 (2011).

36. N. Chaâbane, V. Suendo, H. Vach and P. Roca i Cabarrocas. Appl. Phys. Lett. 88, 203111 (2006).

37. Ning Ning and H. Vach. J. Phys. Chem. A. 114, 3297 (2010).

38. E. A. G. Hamers, A. Foncuberta, C. Niikura, R. Brenot and P. Roca i Cabarrocas. J. Appl.

Phys., 88, 3674 (2000).

39. E.V.Johnson, S. Pouliquen, P.A. Delattre, and J.P. Booth, Plasma Assisted Materials

Processing and Synthesis - 2011 (Mater. Res. Soc. Symp. Proc., San Francisco, April) S4.5.

40. E.V. Johnson, P-A. Delattre, and J.P. Booth, Appl. Phys. Lett. 100, 133504 (2012).

41. A. Fontcuberta i Morral, P. Roca i Cabarrocas and C. Clerc. Phys. Rev. B 69, 125307 (2004).

42. T. Takagi, Thin Solid Films 92, 1 (1982).

43. R. Biswas, G. S. Grest, and C.M. Soukoulis. Phys. Rev. B 38, 8154 (1988).

44. L. Csepregi, E.F. Kennedy, and J.W. Mayer, J. Appl. Phys. 49, 3906 (1978).

45. H. Vach, Q. Brulin, Phys. Rev. Lett., 95, 165502 (2005). 